\title{
Brain Imaging Studies in Pathological Gambling
}

\author{
Ruth J. van Holst • Wim van den Brink • \\ Dick J. Veltman • Anna E. Goudriaan
}

Published online: 30 July 2010

(C) The Author(s) 2010. This article is published with open access at Springerlink.com

\begin{abstract}
This article reviews the neuroimaging research on pathological gambling (PG). Because of the similarities between substance dependence and PG, PG research has used paradigms similar to those used in substance use disorder research, focusing on reward and punishment sensitivity, cue reactivity, impulsivity, and decision making. This review shows that PG is consistently associated with blunted mesolimbic-prefrontal cortex activation to nonspecific rewards, whereas these areas show increased activation when exposed to gambling-related stimuli in cue exposure paradigms. Very little is known, and hence more research is needed regarding the neural underpinnings of impulsivity and decision making in PG. This review concludes with a discussion regarding the challenges and new developments in the field of neurobiological gambling research and comments on their implications for the treatment of PG.
\end{abstract}

Keywords Pathological gambling · Addiction .

Neuroimaging $\cdot$ Neuropsychology

\section{Introduction}

When gambling behavior becomes compulsive, starts to interfere with relationships, and negatively affects social activities or work, it is defined as pathological gambling (PG). Although PG is classified as an impulse control disorder in the DSM-IV, it is often regarded as a behavioral

R. J. van Holst $(\bowtie) \cdot$ W. van den Brink • D. J. Veltman •

A. E. Goudriaan

Department of Psychiatry, Academic Medical Center,

University of Amsterdam,

P. O. Box 22660, 1100 DD Amsterdam, The Netherlands

e-mail: r.j.vanholst@amc.uva.nl or nonchemical addiction because of its genetic, endophenotypic, and phenotypic resemblances to substance dependence. For example, diagnostic criteria for PG resemble those of substance dependence, and both disorders show similar comorbidity patterns [1], genetic vulnerabilities, and responses to specific pharmacologic treatments [2].

Investigating PG as a model of addictive behavior is attractive because it may reveal how addictive behaviors can develop and affect brain function, without the confounding effects of (neurotoxic) substances. Moreover, better understanding of the neurobiological basis of PG could help improve treatment for this disorder.

Given the similarities between PG and substance dependence, $\mathrm{PG}$ research has made assumptions and used paradigms similar to those used in substance use disorder (SUD) research. Current addiction theories have identified four important cognitive-emotional processes that are likely to be relevant for PG as well. The first of these is reward and punishment processing and its relation to behavioral conditioning. The second process is increased salience of gambling cues that often results in strong urges or craving for gambling. The third is impulsivity because it has been implicated as a vulnerability trait for acquiring PG and as a consequence of gambling problems. The fourth process is impaired decision making because pathological gamblers continue gambling in the face of severe negative consequences.

Although neuropsychological studies in PG have consistently reported aberrant function in these domains [3, $4 \cdot \bullet$ ], implementation of neuroimaging techniques has only recently begun to elucidate the neurobiology of PG. In this review, neuroimaging findings in PG are discussed using the four processes just described as an organizing principle.

Based on the search criteria used in the recent review of van Holst et al. [4••], which included 10 neuroimaging studies published since 2005, we updated this selection 
with three studies published or submitted since that review (ie, 2009-2010). Furthermore, we discuss challenges and novel developments in the field of neurobiological gambling research and comment on their implications for the treatment of PG.

\section{Reward and Punishment Sensitivity}

Behavioral conditioning is a key process involved in the development of gambling behavior because gambling operates on a variable intermittent pattern of reinforcement [5]. Differences in behavioral conditioning depend on underlying reward and punishment sensitivity, which have been studied in PG relatively often with neuroimaging techniques.

Reuter et al. [6] compared functional MRI (fMRI) blood oxygen level dependence (BOLD) responses associated with reward and punishment events in 12 pathological gamblers and 12 normal controls (NCs) using a guessing paradigm. They reported lower ventral striatal and ventromedial prefrontal cortex (VMPFC) activity in pathological gamblers when they were receiving monetary gains compared with controls. Comparable results were reported in a study by de Ruiter et al. [7•], who used an affective switching paradigm to investigate the effects of reward and punishment on subsequent behavior. Imaging data associated with monetary gains showed that pathological gamblers $(n=19)$ had lower ventrolateral prefrontal cortex activation to monetary gain than NCs $(n=19)$. In addition, this study showed lower sensitivity to monetary losses in pathological gamblers than among NCs. Whereas Reuter et al. [6] found differences predominantly in ventromedial portions of the prefrontal cortex, de Ruiter and colleagues [7•] reported differences mainly in ventrolateral prefrontal regions. In their discussion, de Ruiter et al. [7•] suggested that their lack of VMPFC findings was probably a result of signal loss caused by tissue inhomogeneity in these regions.

Thus, pathological gamblers were found to have diminished ventral striatum and ventral prefrontal activation during nonspecific rewarding and punishing events compared with NCs [6, 7•], implicating a blunted neurophysiologic response to rewards as well as to losses in pathological gamblers. The reported diminished ventral striatum activation in response to nonspecific rewarding and punishing events found by Reuter et al. [6] is similar to findings in SUDs $[8,9]$. Moreover, most addiction theories have stated that substance dependence is characterized by decreased basal ganglia dopaminergic transmission predating the development of addictive behavior, and that repeated drug use results in a further reduction of dopamine (DA) transmission associated with diminished sensitivity to rewarding stimuli [10]. In line with these theories, it has been hypothesized that pathological gamblers are more likely to seek rewarding events to compensate for a preexisting anhedonic state comparable with that of substance-dependent individuals [11]. However, from the existing literature on $\mathrm{PG}$, it is not yet clear whether diminished reward and punishment sensitivity is a consequence or a precursor of problem gambling.

\section{Cue Reactivity}

In addition to reward system dysfunction, a prominent symptom of PG is the strong urge to gamble, which often leads to a relapse in gambling behavior. Although craving and cue reactivity have been extensively studied with neuroimaging techniques in SUDs, only a few studies in PG have been published.

The first fMRI study on gambling urges was published in 2003 [12]. While viewing a gambling video designed to evoke emotional and motivational antecedents to gambling (actors who mimicked emotional [eg, happy, angry] situations followed by the actor describing driving to and walking through a casino and the feeling of gambling), participants were asked to press a button when they experienced gambling urges. During such episodes of increased craving, the PG group $(n=10)$ showed less activation in the cingulate gyrus, (orbito)frontal cortex, caudate, basal ganglia, and thalamic areas compared with the $\mathrm{NC}$ group $(n=11)$. Recently, the authors reanalyzed their 2003 data to determine whether motivational processing in pathological gamblers $(n=10)$ and cocaine users $(n=9)$ differed from that of recreational gamblers $(n=11)$ and NCs $(n=6)$ not using cocaine [13]. Viewing of addiction-related scenarios compared with neutral scenarios resulted in increased activity in the ventral and dorsal anterior cingulate cortex and right inferior parietal lobule, with relatively decreased activity in pathological gamblers compared with recreational gamblers, and relatively increased activity in cocaine users compared with NCs. These findings therefore indicate opposite effects in individuals with an SUD compared with those with a behavioral addiction.

In contrast, an fMRI cue reactivity study by Crockford et al. [14] found a higher BOLD response in the right dorsolateral prefrontal cortex (DLPFC), right inferior frontal gyrus, medial frontal gyrus, left parahippocampal region, and left occipital cortex in response to gambling stimuli in pathological gamblers $(n=10)$ compared with NCs $(n=11)$. In addition, the dorsal visual processing stream was activated in pathological gamblers when they were viewing gambling movies, whereas the ventral visual stream was activated in controls when they viewed these movies. The authors argued that brain regions activated in pathological gamblers compared with NCs predominantly 
involved regions associated with the DLPFC network, which is associated with conditional responses.

In a recent study, Goudriaan et al. [15] showed similar cue reactivity-related brain activations as reported by Crockford et al. [14] in pathological gamblers $(n=17)$ compared with NCs $(n=17)$. In this fMRI study, participants viewed gambling pictures and neutral pictures while being scanned. When viewing gambling pictures versus neutral pictures, higher bilateral parahippocampal gyrus, right amygdala, and right DLPFC activity was found in problem gamblers relative to NCs. Furthermore, a positive relationship was found between subjective craving for gambling after scanning in problem gamblers and BOLD activation in the ventrolateral prefrontal cortex, left anterior insula, and left caudate head when viewing gambling pictures versus neutral pictures.

Finally, in a recent gambling paradigm study, 12 problems gamblers and 12 frequent (nonproblem) gamblers were asked to play a blackjack gambling game while fMRI scans were obtained [16]. The game consisted of trials with a high risk of losing and trials with a low risk of losing. Problem gamblers showed a signal increase in thalamic, inferior frontal, and superior temporal regions during high-risk trials and a signal decrease in these regions during low-risk trials, whereas the opposite pattern was observed in frequent gamblers. Miedl and colleagues [16] argued that the frontal-parietal activation pattern noted during high-risk trials compared with low-risk trials in problem gamblers reflects a cue-induced addiction memory network that is triggered by gambling-related cues. They suggested that high-risk situations might serve as an addiction cue in problem gamblers, whereas the low-risk situation signifies a "safe" hit in frequent gamblers. Interestingly, problem gamblers showed higher activity in dorsolateral prefrontal and parietal lobes compared with frequent gamblers while winning as compared with losing money, a network generally associated with executive function. However, activity patterns in limbic regions while winning compared with losing money were similar, which is at odds with earlier findings of reward processing in the studies by Reuter et al. [6] and de Ruiter et al. [7•]. Differences in the employed paradigms could explain the dissimilarities between these studies: whereas in the blackjack paradigm of Miedl and colleagues [16], the winning result had to be calculated by the participants (calculating the card values) before they realized that a win or loss was experienced, in the studies by Reuter et al. [6] and de Ruiter et al. [7•], wins or losses were displayed on the screen and thus experienced immediately. Therefore, in the study by Miedl et al. [16], the relatively high stimulus complexity and cognitive elements in reward and loss experiencing may have influenced reward processing and diminished the potential to find group differences.

Thus, cue reactivity studies in PG have so far reported conflicting results. It should be noted, however, that the findings of Potenza et al. [12, 13] are difficult to interpret because of the complex emotional movies used to elicit craving for gambling. On the other hand, the increased activity in response to gambling cues in the prefrontal cortex, parahippocampal regions, and occipital cortex reported by Crockford et al. [14], Goudriaan et al. [15], and Miedl et al. [16] is consistent with results from cue reactivity paradigms in SUD studies [17, 18]. However, in contrast to SUD studies, enhanced limbic activation during cue reactivity paradigms in gambling was only reported in one of the gambling cue reactivity studies [15]. Future research should focus on the type of stimuli that elicit the most powerful cue reactivity (eg, pictures vs movies). One aspect that may diminish the power to detect differences in cue reactivity in PG studies as opposed to SUD studies is that gambling may involve a diversity of gambling activities (eg, blackjack, slot machines, horse racing), whereas cue reactivity to a substance is more specific for the targeted substance (eg, cocaine, marijuana) and may therefore elicit limbic brain activity in most SUD participants. Selecting specific gambling types for cue reactivity stimuli and limiting participant inclusion to a specific gambling pathology may result in a better matching of cues and PG pathology and thus result in more robust brain activations in response to cues in PG.

\section{Impulsivity in Pathological Gambling}

Impulsivity is often equated with disinhibition, a state during which top-down control mechanisms that normally suppress automatic or reward-driven responses are inadequate to meet current demands [19]. Disinhibition has received considerable attention in addiction research in recent years because it has been recognized as an endophenotype of individuals at risk of SUD and PG [20]. Another aspect of impulsivity that is frequently addressed in neurocognitive studies is delay discounting: choosing for immediate smaller rewards instead of delayed larger rewards. This aspect is discussed in the next section on decision making. Unfortunately, neuroimaging studies investigating the neural correlates of impulsivity/ disinhibition in PG are scarce.

In the only fMRI study published to date, Potenza et al. [21] used a Stroop color-word task to assess cognitive inhibition - that is, inhibition of an automatic response (congruent stimulus; reading a word) compared with naming the color in which the word is printed (incongruent stimulus) - in 13 pathological gamblers and 11 NCs. Pathological gamblers showed lower activation in the left middle and superior frontal gyri compared with the $\mathrm{NC}$ group during processing of incongruent versus congruent stimuli.

In summary, although several neuropsychological studies have indicated higher impulsivity in pathological 
gamblers [22, 23], to date, only a single neuroimaging study on inhibition has been published. Therefore, additional neuroimaging studies are warranted, preferably with larger populations and assessment of a variety of impulsivity measures in pathological gamblers.

\section{Decision Making in Pathological Gambling}

Pathological gamblers and SUD patients exhibit a pattern of decision making characterized by ignoring long-term negative consequences to obtain immediate gratification or relief from uncomfortable states associated with their addiction [24]. A variety of cognitive and emotional processes may affect decision making. Risk taking, experiencing and evaluating immediate versus delayed wins and losses, and impulsivity have been found to contribute to the multifaceted concept of decision making [25]. In addition, executive dysfunctionsmainly diminished cognitive flexibility-have been associated with impairments in decision making [26].

In a recent event-related potential (ERP) study [27], neurophysiologic correlates of decision making during a blackjack game were measured. Twenty problem gamblers and 21 NCs played a computerized blackjack game and had to decide if they would "hit" or "sit" a card to arrive as close as possible to, but not greater than 21 points. At the critical score of 16 points, problem gamblers decided more often than NCs to continue playing. Furthermore, problem gamblers showed greater positive amplitude in the ERPs, modelled by a dipole in the anterior cingulate cortex, than NCs after successful "hit" decisions at 16. Thus, gamblers showed more risk-taking behavior coupled with a stronger neural response to (infrequent) successful outcomes of this behavior compared with NCs. Interestingly, no neurophysiologic differences were observed between groups during loss trials.

Until now, no other neuroimaging studies focusing on decision-making processes in pathological gamblers have been published. However, one fMRI study used a modified version of the Iowa Gambling Task (IGT) to investigate decision-making performance in NCs $(n=16)$, individuals with substance dependence (SD; $n=20)$ and substancedependent individuals with comorbid gambling problems (SDPG; $n=20$ ) [28]. The IGT was created to mimic real life decision making [29]. Participants were presented with four virtual decks of cards on a computer screen from which they had to choose a card. Every card drawn would result in a reward, but occasionally, a card would result in a loss. Hence, some decks would lead to losses in the long run, and others would lead to gains. The goal of the game was to win as much money as possible. Although SDPGs tended to perform better than SDs and NCs, these differences were not statistically significant. SD and SDPG individuals showed lower VMPFC activity compared with NCs when performing the IGT. Furthermore, the SD group showed less right superior frontal cortex activity during decision making than the SDPG and NC groups. The authors concluded that greater right superior frontal cortex activity in SDPGs compared with SDs may reflect hypersensitivity to gambling cues, because the IGT resembles a gambling game. Unfortunately, the study did not include a pathological gambler group without comorbid SUDs. These results suggest that comorbid $\mathrm{PG}$ is not associated with an added impairment in decision making in SD, a finding inconsistent with a neurocognitive study of pathological gamblers, SUDs, and NCs [23]. These incongruent findings could be explained by the fact that Tanabe et al. [28] used a modified version of the IGT that prevented successive choices from a particular deck, thereby facilitating correct choices in the SD groups by eliminating the need for cognitive flexibility that is likely to be defective in pathological gamblers [26, 30].

\section{Conclusions}

The reviewed studies indicate that pathological gamblers show decreased BOLD responses to nonspecific rewarding and punishing stimuli in the ventral striatum and VMPFC $[6,7 \cdot]$. Notably, such blunted responses were not observed in problem gamblers playing a more realistic gambling game during the winning and losing of money [16]. Three of four neuroimaging studies on cue reactivity in pathological gamblers showed increased brain activation to gambling-related stimuli [14-16], whereas results from the other study, which reported diminished brain activation during a craving paradigm, were difficult to interpret due to the complex stimulus paradigm used [12, 13]. The neurobiological mechanisms underlying abnormal cue reactivity in pathological gamblers are therefore not yet clear, and the same is true for the observed increased impulsivity and disinhibition in pathological gamblers. In addition, whereas a large number of neurocognitive studies on impulsivity have indicated that pathological gamblers are impaired in several inhibitory processes (eg, filtering irrelevant information, inhibiting ongoing responses, and delay discounting [4••]), to date, only one fMRI study on Stroop interference in pathological gamblers has been published [21]. Similarly, although neurocognitive studies have indicated impaired decision making among pathological gamblers [4••], which is consistent with findings in substance dependence [31], only one ERP study on decision making in pathological gamblers is currently available [27]. This latter study indicated that problem gamblers displayed more risktaking behavior during gambling than $\mathrm{NCs}$, and that 
successful but risky decisions were associated with greater activity in the anterior cingulate cortex. Finally, an fMRI study investigating decision making using the IGT indicated lower superior frontal cortex activity during decision making in substance-dependent individuals with gambling problems.

\section{Clinical Implications}

Although the overall number of neuroimaging studies in pathological gamblers is still modest, fMRI studies have consistently shown diminished activity in the mesolimbic pathways in pathological gamblers comprising the ventral striatum, amygdala, and VMPFC when problem gamblers are dealing with reward and loss processing, but not when they are in a gambling situation. These brain circuits are thought to play an important role in integrating emotional processing and behavioral consequences in healthy individuals. Because the VMPFC depends on DA projections from other limbic structures to integrate information, impaired DA transmission may underlie VMPFC dysfunction in pathological gamblers. However, many other neurotransmitter systems are probably also engaged and may interact during the processing of positive and negative feedback. For example, opiates are known to increase DA release in the brain reward pathways, and opiate antagonists that decrease dopamine release (eg, naltrexone and nalmefene), have been found to reduce reward sensitivity and probably increase punishment sensitivity [32]. This may be the reason why opiate antagonists are more effective in treating PG than placebo [33]. The effectiveness of opiate antagonists indicates that targeting the reward system of the brain may be a fruitful strategy in battling craving urges in PG, similar to studies in alcohol and amphetamine dependence [34]. Correspondingly, pharmacologic agents modulating glutamate function (eg, N-acetylcysteine) with known effects on the reward system also have been effective in reducing gambling behavior in pathological gamblers [35].

Impulsivity and impaired impulse control have been targeted by selective serotonin reuptake inhibitors (SSRIs) in impulse control disorders [36]. SSRI treatment has yielded mixed results in pathological gamblers [36]. However, the presence or absence of a comorbid condition often may shape the effectiveness of medication used to treat PG. Whereas SSRIs such as fluvoxamine may be effective in treating pathological gamblers with comorbid depression or an obsessive-compulsive spectrum disorder, they may not be the treatment of choice in pathological gamblers with comorbid attention-deficit/hyperactivity disorder. Medications to improve decision making and executive function are less well-established, probably because of the complexity of these functions. Therefore, the potential efficacy of cognitive enhancers such as modafinil will have to be substantiated in future PG medication studies [37]. Cognitive-behavioral therapy is also effective in treating PG [38]. Future research should clarify whether a combination of pharmacotherapy and psychological treatment will lead to more sustained remission rates in PG than either therapy alone.

\section{Future Directions}

Neurocognitive similarities and comparable pharmacologic responsiveness in PG and SUDs seem to point to a common vulnerability to addictive behaviors, and perhaps similar pathological pathways underlying PG and SUDs. These similarities provide a rationale to change the classification of PG as an impulse control disorder to a new classification of $\mathrm{PG}$ as a behavioral addiction in the DSM- $V$. However, more research is needed to elucidate which neurocognitive similarities and differences exist between SUDs and PG, and studies directly comparing these disorders to each other and to NC groups are evidently needed.

Furthermore, similar to methods used in SUD research, future PG research combining pharmacologic challenges with neuroimaging techniques may aid in unravelling the neurobiological mechanisms of PG. For example, naltrexone could be used to manipulate opiate function in an fMRI study on reward and punishment sensitivity, cue reactivity, and craving.

Using "state-of-the-art" neuromodulation techniques such as repetitive transcranial magnetic stimulation (rTMS) could further illustrate the involvement of various brain regions found in fMRI paradigms in gambling behavior. For example, the key role of the DLPFC in preventing relapse behavior was supported by an rTMS study demonstrating that high-frequency DLPFC stimulation in former smokers resulted in lower relapse rates and craving for smoking compared with former smokers who received sham rTMS [39]. Moreover, rTMS of the prefrontal cortex was shown to change prefrontal function in addictive disorders [40], although long-term effects on relapse are less well-established. Using such designs could inform us about localization of brain functions critically involved in addictive behavior and eventually offer new treatment options for PG.

Another interesting approach is the application of neurofeedback in PG. By training individuals to change specific brain activity patterns, we can test to determine how this affects gambling behavior. This technique has already been implemented in the treatment of attentiondeficit/hyperactivity disorder [41] and could be effective in PG as well. For example, studies have indicated abnormal prefrontal function in PG $[6,7 \bullet, 21]$, and neurofeedback training can be focused on normalizing frontal electroen- 
cephalogram patterns. By targeting focal prefrontal function, executive functions may be trained, which may result in improved cognitive control and, hence, diminished likelihood of relapse when craving occurs.

Interestingly, a growing number of studies have reported the development of PG during treatment of Parkinson's disease (PD). PD is characterized by loss of dopaminergic neurons in the mesolimbic and mesocortical networks, and treatment with DA agonists has been associated with reward-seeking behaviors such as PG, compulsive shopping, and disinhibition [42]. These behaviors likely reflect modulation of reward circuitry functions by dopaminergic drugs. Neuroimaging studies have reported decreased activation in the mesolimbic pathway during monetary gains in PD [43], similar to findings in PG and other addictions. In addition, lower D2/D3 binding was reported in a positron emission tomography study in PD with comorbid PG compared with a control group with PD only [44•]. Furthermore, Eisenegger et al. [45•] found that healthy individuals who carry at least one copy of the 7repeat DRD4 DA receptor allele showed an increased gambling propensity after dopaminergic stimulation with L-DOPA. These findings demonstrate that genetic variation in the $D R D 4$ gene can determine an individual's gambling behavior in response to a dopaminergic drug challenge. These observations are consistent with a reward deficiency syndrome [46]. This postulates a chronic hypodopaminergic state rendering individuals vulnerable to addictions by triggering a drive for rewarding substances or behaviors to boost low dopaminergic activity in the brain's reward circuitry. Future research investigating dopaminergic dysregulation and interactions with genetic variations in PD patients with and without PG may contribute to our understanding of neurophysiologic factors predisposing individuals to addictive behaviors.

Additional studies are similarly needed to investigate expectancy values in pathological gamblers to explain abnormal reward and punishment sensitivity, as these abnormalities could be related to aberrant expectations rather than to the actual experiences of reward and loss. For example, a gambler may become biased in his or her expectations of chances of winning because being in a gambling situation provokes cue reactivity in the brain, heightening DA release in the mesolimbic circuit. The associated enhanced DA signalling could trigger a disruption of the correct expectancy coding because phasic DA changes are crucial for expectancy coding [47]. Thus, by enhanced cue reactivity, expectations are erroneously coded and could contribute to continuation of gambling despite heavy losses. In addition, abnormal expectancy values could be influenced by cognitive distortions, such as erroneous beliefs regarding the probability of winning [48].
Gambling games are thought to foster certain features that may exaggerate confidence of one's chances of winning, thereby stimulating gambling propensity. In a recent fMRI study, Clark et al. [49••] investigated two of these characteristics: personal control over the game and the "near-win" event in NCs. Near-win events are events in which unsuccessful outcomes are proximal to the jackpot, such as when two cherries are displayed on the slot machine pay line, and the last cherry ends up one position below or above the pay line. Interestingly, nearwin outcomes activated ventral striatal and insula regions that also responded to monetary wins. Such findings may provide insights into the underlying mechanisms responsible for the continuation of gambling behavior in spite of the notion that one will lose money over time. Future research should elaborate on these findings to help us further understand the transition of gambling to problem gambling and the addictive potential of certain game characteristics.

A final area for future development is the subject of resistance to the development of addictive behaviors. Blaszczynski and Nower [5] described a class of problem gamblers without comorbidities and minimal pathology. This less severe gambling group was also thought to be able to overcome their gambling problems without therapeutic interventions. Studying different subgroups of pathological gamblers may yield insight into the neuropsychological functions that are protective against the progression of problem gambling and/or against relapse. The neurobiological factors that are clearly involved in PG and that may influence the course of PG are executive functions, including decision making and impulsivity; cue reactivity; reward sensitivity; and erroneous perceptions. From the review of neuroimaging studies, it is clear that the neuronal background of these functions has not yet been identified in detail. However, these neurobiological vulnerabilities are likely to influence the course of PG in combination with psychological factors, such as subjective craving and coping skills; environmental factors (eg, the vicinity of gambling opportunities); and genetic factors. How these factors interact is largely unknown. Understanding these phenomena and their interactions is of great importance because interventions focusing on these vulnerabilities could ultimately lead to targeted prevention measures.

Acknowledgments Ruth J. van Holst is supported by a neuroimaging grant from the Amsterdam Brain Imaging Platform. Dr. Goudriaan is supported by a new investigator grant (Veni grant no. 91676084) from the Netherlands Organization for Health Research and Development.

Disclosure No potential conflicts of interest relevant to this article were reported. 
Open Access This article is distributed under the terms of the Creative Commons Attribution Noncommercial License which permits any noncommercial use, distribution, and reproduction in any medium, provided the original author(s) and source are credited.

\section{References}

Papers of particular interest, published recently, have been highlighted as:

- Of importance

-. Of major importance

1. Petry NM, Stinson FS, Grant BF: Comorbidity of DSM-IV pathological gambling and other psychiatric disorders: results from the National Epidemiologic Survey on Alcohol and Related Conditions. J Clin Psychiatry 2005, 66:564-574.

2. Petry NM: Gambling and substance use disorders: current status and future directions. Am J Addict 2007, 16:1-9.

3. Goudriaan AE, Oosterlaan J, de Beurs E, et al.: Pathological gambling: a comprehensive review of biobehavioral findings. Neurosci Biobehav Rev 2004, 28:123-141.

4. •- van Holst RJ, van den Brink W, Veltman DJ, Goudriaan AE: Why gamblers fail to win: a review of cognitive and neuroimaging findings in pathological gambling. Neurosci Biobehav Rev 2010, 34:87-107. In this recent review, the findings of neurocognitive research in PG from 2005 to 2009 are described and discussed in detail. The results indicate that $P G$ is more than an impulse control disorder and that neurocognitive findings fit well with theoretical models of addiction. We also discuss potential fruitful research areas concerning $P G$ to help us further understand this disorder.

5. Blaszczynski A, Nower L: A pathways model of problem and pathological gambling. Addiction 2002, 97:487-499.

6. Reuter J, Raedler T, Rose M, et al.: Pathological gambling is linked to reduced activation of the mesolimbic reward system. Nat Neurosci 2005, 8:147-148.

7. - de Ruiter MB, Veltman DJ, Goudriaan AE, et al.: Response perseveration and ventral prefrontal sensitivity to reward and punishment in male problem gamblers and smokers. Neuropsychopharmacology 2009, 34:1027-1038. In this fMRI study, it was shown that problem gamblers have a blunted BOLD response during reward or loss processing. These findings are similar to findings in substance-dependent individuals tested with paradigms probing the reward system. These findings are in line with addiction theories postulating that a decreased dopaminergic transmission predates the development of addictive behavior, and that repeated drug use-or gambling - results in a further reduction of DA transmission associated with diminished sensitivity to rewarding stimuli.

8. Heinz A, Wrase J, Kahnt T, et al.: Brain activation elicited by affectively positive stimuli is associated with a lower risk of relapse in detoxified alcoholic subjects. Alcohol Clin Exp Res 2007, 31:1138-1147.

9. Wrase J, Schlagenhauf F, Kienast T, et al.: Dysfunction of reward processing correlates with alcohol craving in detoxified alcoholics. Neuroimage 2007, 35:787-794.

10. Goldstein RZ, Volkow ND: Drug addiction and its underlying neurobiological basis: neuroimaging evidence for the involvement of the frontal cortex. Am J Psychiatry 2002, 159:1642-1652.

11. Robinson TE, Berridge KC: Review. The incentive sensitization theory of addiction: some current issues. Philos Trans R Soc Lond B Biol Sci 2008, 363:3137-3146.
12. Potenza MN, Steinberg MA, Skudlarski P, et al.: Gambling urges in pathological gambling: a functional magnetic resonance imaging study. Arch Gen Psychiatry 2003, 60:828-836.

13. Potenza MN: Review. The neurobiology of pathological gambling and drug addiction: an overview and new findings. Philos Trans R Soc Lond B Biol Sci 2008, 363:3181-3189.

14. Crockford DN, Goodyear B, Edwards J, et al.: Cue-induced brain activity in pathological gamblers. Biol Psychiatry 2005, 58:787795.

15. Goudriaan AE, de Ruiter MB, van den Brink W, et al.: Brain activation patterns associated with cue reactivity and craving in abstinent problem gamblers, heavy smokers and healthy controls: an fMRI study. Addict Biol 2010 (in press).

16. Miedl SF, Fehr T, Meyer G, et al.: Neurobiological correlates of problem gambling in a quasi-realistic blackjack scenario as revealed by fMRI. Psychiatry Res 2010, 181:165-173.

17. George MS, Anton RF, Bloomer C, et al.: Activation of prefrontal cortex and anterior thalamus in alcoholic subjects on exposure to alcohol-specific cues. Arch Gen Psychiatry 2001, 58:345-352.

18. Wrase J, Grusser SM, Klein S, et al.: Development of alcoholassociated cues and cue-induced brain activation in alcoholics. Eur Psychiatry 2002, 17:287-291.

19. Aron AR: The neural basis of inhibition in cognitive control. Neuroscientist 2007, 13:214-228.

20. Verdejo-Garcia A, Lawrence AJ, Clark L: Impulsivity as a vulnerability marker for substance-use disorders: review of findings from high-risk research, problem gamblers and genetic association studies. Neurosci Biobehav Rev 2008, 32:777-810.

21. Potenza MN, Leung HC, Blumberg HP, et al.: An FMRI Stroop task study of ventromedial prefrontal cortical function in pathological gamblers. Am J Psychiatry 2003, 160:1990-1994.

22. Goudriaan AE, Oosterlaan J, de Beurs E, et al.: Neurocognitive functions in pathological gambling: a comparison with alcohol dependence, Tourette syndrome and normal controls. Addiction 2006, 101:534-547.

23. Petry NM: Substance abuse, pathological gambling, and impulsiveness. Drug Alcohol Depend 2001, 63:29-38.

24. Yechiam E, Busemeyer JR, Stout JC, et al.: Using cognitive models to map relations between neuropsychological disorders and human decision-making deficits. Psychol Sci 2005, 16:973978.

25. Krawczyk DC: Contributions of the prefrontal cortex to the neural basis of human decision making. Neurosci Biobehav Rev 2002, 26:631-664.

26. Clark L, Cools R, Robbins TW: The neuropsychology of ventral prefrontal cortex: decision-making and reversal learning. Brain Cogn 2004, 55:41-53.

27. Hewig J, Kretschmer N, Trippe RH, et al.: Hypersensitivity to reward in problem gamblers. Biol Psychiatry 2010, 67:781783.

28. Tanabe J, Thompson L, Claus E, et al.: Prefrontal cortex activity is reduced in gambling and nongambling substance users during decision-making. Hum Brain Mapp 2007, 28:1276-1286.

29. Bechara A, Damasio H, Tranel D, et al.: Deciding advantageously before knowing the advantageous strategy. Science 1997, 275:1293-1295.

30. Brand M, Kalbe E, Labudda K, et al.: Decision-making impairments in patients with pathological gambling. Psychiatry Res 2005, 133:91-99.

31. Dom G, De Wilde B, Hulstijn W, et al.: Decision-making deficits in alcohol-dependent patients with and without comorbid personality disorder. Alcohol Clin Exp Res 2006, 30:1670-1677.

32. Petrovic P, Pleger B, Seymour B, et al.: Blocking central opiate function modulates hedonic impact and anterior cingulate response to rewards and losses. J Neurosci 2008, 28:10509-10516. 
33. Grant JE, Kim SW, Hartman BK: A double-blind, placebo-controlled study of the opiate antagonist naltrexone in the treatment of pathological gambling urges. J Clin Psychiatry 2008, 69:783-789.

34. O'Brien CP: Anticraving medications for relapse prevention: a possible new class of psychoactive medications. Am J Psychiatry 2005, 162:1423-1431.

35. Grant JE, Kim SW, Odlaug BL: N-acetyl cysteine, a glutamatemodulating agent, in the treatment of pathological gambling: a pilot study. Biol Psychiatry 2007, 62:652-657.

36. Hollander E, Sood E, Pallanti S, et al.: Pharmacological treatments of pathological gambling. J Gambl Stud 2005, 21:99-110.

37. Minzenberg MJ, Carter CS: Modafinil: a review of neurochemical actions and effects on cognition. Neuropsychopharmacology 2008, 33:1477-1502.

38. Petry NM, Ammerman Y, Bohl J, et al.: Cognitive-behavioral therapy for pathological gamblers. J Consult Clin Psychol 2006, 74:555-567.

39. Amiaz R, Levy D, Vainiger D, et al.: Repeated high-frequency transcranial magnetic stimulation over the dorsolateral prefrontal cortex reduces cigarette craving and consumption. Addiction 2009, 104:653-660.

40. Barr MS, Fitzgerald PB, Farzan F, et al.: Transcranial magnetic stimulation to understand the pathophysiology and treatment of substance use disorders. Curr Drug Abuse Rev 2008, 1:328-339.

41. Arns M, de Ridder S, Strehl U, et al.: Efficacy of neurofeedback treatment in ADHD: the effects on inattention, impulsivity and hyperactivity: a meta-analysis. Clin EEG Neurosci 2009, 40:180-189.

42. Torta DM, Castelli L: Reward pathways in Parkinson's disease: clinical and theoretical implications. Psychiatry Clin Neurosci 2008, 62:203-213.

43. Thiel A, Hilker R, Kessler J, et al.: Activation of basal ganglia loops in idiopathic Parkinson's disease: a PET study. J Neural Transm 2003, 110:1289-1301.

44. - Steeves TD, Miyasaki J, Zurowski M, et al.: Increased striatal dopamine release in Parkinsonian patients with pathological gambling: a [11C] raclopride PET study. Brain 2009, 132:1376-
1385. This article reports on an $\left[{ }^{11} C\right]$ raclopride positron emission tomography study comparing DA function during gambling in $P D$ patients, with and without $P G$, following treatment with $D A$ agonists. The authors found lower binding potential, probably reflecting greater dopaminergic release, in pathological gamblers. The emergence of $P G$ in several $P D$ patients treated with $D A$ agonists could serve as a model for the pathophysiology of $P G$.

45. - Eisenegger C, Knoch D, Ebstein RP, et al.: Dopamine receptor D4 polymorphism predicts the effect of L-DOPA on gambling behavior. Biol Psychiatry 2010, 67:702-706. This recent study investigated the relationship between DA agonist treatment in PD and the development of $P G$, focusing on the role of the variation in DA receptor gene DRD4. Although L-DOPA administration did not lead to increased gambling propensity compared with placebo in patients with $P D$, individuals with at least one copy of the 7 repated allele of DRD4 showed increased gambling propensity after administration of $L-D O P A$.

46. Blum K, Braverman ER, Holder JM, et al.: Reward deficiency syndrome: a biogenetic model for the diagnosis and treatment of impulsive, addictive, and compulsive behaviors. J Psychoactive Drugs 2000, 32(Suppl):i-112

47. Schultz W: Behavioral dopamine signals. Trends Neurosci 2007, 30:203-210.

48. Toneatto T, Blitz-Miller T, Calderwood $\mathrm{K}$, et al.: Cognitive distortions in heavy gambling. J Gambl Stud 1997, 13:253-266.

49. •• Clark L, Lawrence AJ, Astley-Jones F, et al.: Gambling nearmisses enhance motivation to gamble and recruit win-related brain circuitry. Neuron 2009, 61:481-490. This recent study investigated the potential neuropsychological mechanisms behind the addictive potential of gambling. Near-miss events, in which unsuccessful outcomes are proximal to the jackpot, were studied with an fMRI paradigm in NCs. Interestingly, near-miss outcomes recruited striatal and insula circuitry that also responded to monetary wins. This study offers insight into the effect of a nearmiss, which invigorates gambling by anomalous recruitment of the reward circuitry. 